\title{
Theory of light amplification in active fishnet metamaterials
}

\author{
Joachim M. Hamm, Sebastian Wuestner, Kosmas L. Tsakmakidis, and Ortwin Hess* \\ Department of Physics, South Kensington Campus, Imperial College, London SW7 2AZ, UK
}

\begin{abstract}
We establish a theory that traces light amplification in an active double-fishnet metamaterial back to its microscopic origins. Based on ab initio calculations of the light/plasmon fields we extract energy rates and conversion efficiencies associated with gain/loss channels directly from Poynting's theorem. We find that for the negative refactive index mode both radiative loss and gain outweigh resistive loss by more than a factor of two, opening a broad window of steady-state amplification (free of instabilities) accessible even when a gain reduction close to the metal is taken into account.
\end{abstract}

PACS numbers: 78.67.Pt, 42.25.Bs, 78.20.Ci, 78.45.+h

Recent advances in metamaterials research and active plasmonics bring with them the prospect for next generation, loss-free optical metamaterial designs [1]. The double-fishnet metamaterial, first demonstrated in the near infrared [2], is a simple nanoplasmonic structure with a broadband effective negative refractive index, the origins of which are now well established [3]. To date it remains the prime example of a metamaterial able to operate at visible wavelengths [4] and serves as a template for novel optical metamaterial designs [5]. In plasmonics an understanding of how losses experienced by surfaceplasmon polaritons (SPPs) and localized plasmons can be overcome by gain media placed adjacent to metals [6$8]$ has had far reaching impact. This is best illustrated by the realization of SPP amplifiers [9] and nano-plasmonic lasers [10] but also by recent developments in optical metamaterials research. In 2010, first evidence has been given that loss compensation in active plasmonic metamaterials is possible: Experimental [11] and theoretical $[12,13]$ absorption/transmission/reflection (ATR) studies on optically pumped gain-enhanced double-fishnet metamaterials showed that gain can overcome the internal dissipative losses while retaining and in fact enhancing the effective negative refractive index response. Further theoretical work has shown that loss compensation is achieved when the gap-SPP becomes undamped [14] causing the resonance linewidth to be limited by scattering losses only $[14,15]$. The physical origin of light amplification in active fishnet metamaterials lies in the complex interaction of the strong local fields of the gapSPP mode with the spatially inhomogeneous inversion generated by a pump process $[12,16]$. Ultimately, to develop loss-management strategies for future practical metamaterials, a theory is required that traces the internal energy flow, inaccessible by experiment, on the nanometer scale.

In this Letter, we present a theoretical framework that allows us to describe the microscopic dynamical processes, which, collectively, make light amplification in active metamaterials possible. We conduct, under realistic assumptions, extensive numerical pump and probe studies and extract together with the macroscopic spectral

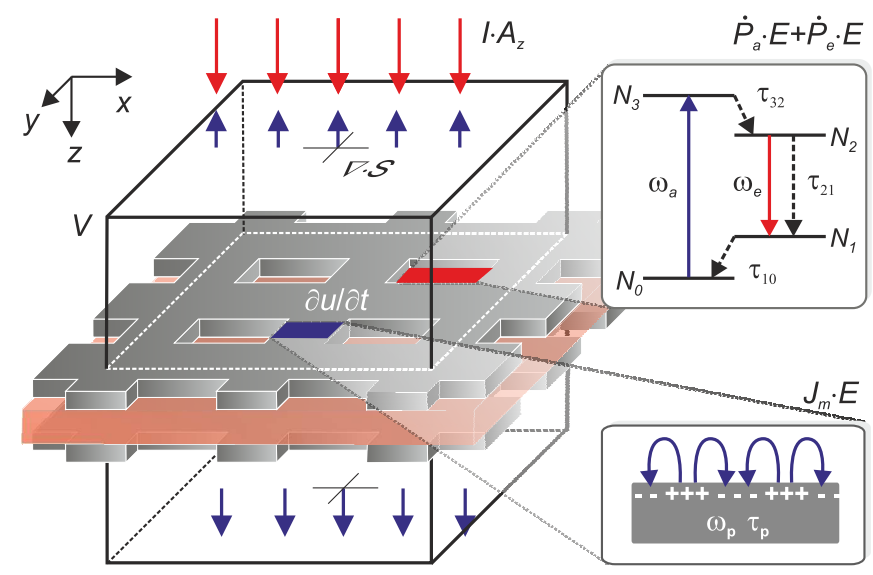

Figure 1. (color online) Schematic of an active plasmonic metamaterial illuminated by an incident plane wave of intensity $I$ that creates a power flux into the volume $V$ through the surface $A_{\mathrm{z}}$. According to Poynting's theorem [see Eq. (1)] the electromagnetic energy $U$ inside the volume changes due to energy transfer into and out of various loss (blue) and gain channels (red). Note that for normal incidence onto an infinite film, the flux between neighbouring volumes cancels.

response the effective rates of energy exchange between physical subsystems of an active fishnet metamaterial. The results demonstrate the intricate mechanisms that make steady-state amplification in nano-plasmonic structures possible: the efficiency of the pump scheme affecting the spatial deposition of inversion, the electric field enhancement that increases the effective gain, and the dominance of radiative coupling over internal losses.

Our studies are based on the Maxwell-Bloch (MB) approach $[15,17]$ combined with a suitable formulation of Poynting's theorem. We solve Maxwell's field equations in full-vectorial three-dimensional form and in interaction with the dynamic polarization responses ascribed to the metal and four-level gain medium microscopically, at every point in space, while simultaneously tracing the flow of electromagnetic energy into and out of microscopic channels (see Fig. 1). This generic method allows us to dynamically record and analyze the gain and loss rates associated with the physical subsystems of any struc- 
ture and thereby to identify transient and steady-state regimes of operation as well as to precisely determine the conditions for amplification and lasing.

We start by recalling Poynting's theorem, according to which the rate of change of the electromagnetic energy inside a volume $V$ that contains metal (loss) and fourlevel gain media is determined by

$$
\langle\partial u / \partial t\rangle=-\langle\nabla \cdot \mathbf{S}\rangle-\left\langle\dot{\mathbf{P}}_{\mathrm{f}} \cdot \mathbf{E}\right\rangle-\left\langle\dot{\mathbf{P}}_{\mathrm{a}} \cdot \mathbf{E}\right\rangle-\left\langle\dot{\mathbf{P}}_{\mathrm{e}} \cdot \mathbf{E}\right\rangle
$$

where $u(\mathbf{r}, t)=\frac{1}{2}\left[\varepsilon_{0} \varepsilon(\mathbf{r}) E^{2}(\mathbf{r}, t)+\mu_{0} H^{2}(\mathbf{r}, t)\right]$ is the energy density, $\mathbf{S}(\mathbf{r}, t)$ the Poynting vector, $\mathbf{E}(\mathbf{r}, t)$ the electric field, and $\mathbf{P}_{i}(\mathbf{r}, t)$ the polarizations of the free electron plasma in the metal $(i=\mathrm{f})$ and the gain media at the transitions of absorption and emission $(i=\mathrm{a}, \mathrm{e})$. The operator $\langle\bullet\rangle$ performs an integration over $V$ and a time-averaging over $\Delta t \gtrsim 2 \pi / \omega$ eliminating fast phase oscillations. Equation (1) expresses the change of electromagnetic energy in $V$ as influx through the surface minus the work that the field exercises on the electrons in the metal and on the transitions of the gain media.

It will prove useful to write Eq. (1) as a rate equation:

$$
\partial U / \partial t=-\Gamma_{\mathrm{t}} U=-\Lambda U-\Gamma_{\mathrm{f}} U-\Gamma_{\mathrm{a}} U-\Gamma_{\mathrm{e}} U
$$

where $U=\left\langle u+\sum_{i} w_{i}\right\rangle$ is the total field energy in the volume, $\Gamma_{\mathrm{t}}$ the energy decay rate, $\Lambda=\langle\nabla \cdot \mathbf{S}\rangle / U$ the flux rate that measures the outflow of energy, and $\Gamma_{\mathrm{i}}=\left[\left\langle\dot{\mathbf{P}}_{\mathrm{i}} \cdot \mathbf{E}-\partial w_{i} / \partial t\right\rangle\right] / U$ the power loss/gain rates. $w_{i}$ are contributions of the dynamic responses to the overall electromagnetic energy in the medium that can be calculated as in [18]. Note that the so-defined effective rates are time-dependent quantities and become constant when $U$ is either constant or decreases exponentially during single mode decay. We also emphasize that this formulation of volume-averaged energy transfer rates is exact (since it directly reflects energy conservation) and applicable to arbitrary linear and nonlinear systems.

The object of our studies is a $260 \mathrm{~nm}$ thick fishnet metamaterial consisting of two 40 nm-thick silver films, separated by $60 \mathrm{~nm}$ and embedded in an active dielectric host of refractive index $n=1.62$ (see Fig. 1). The silver films are perforated with rectangular holes of dimensions $a_{x}=120 \mathrm{~nm}$ and $a_{y}=80 \mathrm{~nm}$ with square periodicity $p=280 \mathrm{~nm}$. For the gain medium (dye) we assume a density of $N=3.89 \times 10^{18} \mathrm{~cm}^{-3}$, wavelength of maximum absorption (emission) $\lambda=680 \mathrm{~nm}(710 \mathrm{~nm})$, and associated cross-section of $\sigma=7.45 \times 10^{-16} \mathrm{~cm}^{2}$ $\left(5.78 \times 10^{-16} \mathrm{~cm}^{2}\right)$. To model the response of silver we use a three-pole Drude-Lorentz model [19].

In our analysis, we first pump the active structure with a pulse of $\lambda_{\mathrm{p}}=680 \mathrm{~nm}$ and then probe it with a signal pulse of center-wavelength $\lambda_{\mathrm{s}}=710 \mathrm{~nm}$. Both the pump and the probe fields are $x$-polarized, and the probe falls within the negative-index resonance of the structure (see Fig. 4 later on). Fabrication tolerances, passivation, and quenching may lead to a reduction of the local gain close
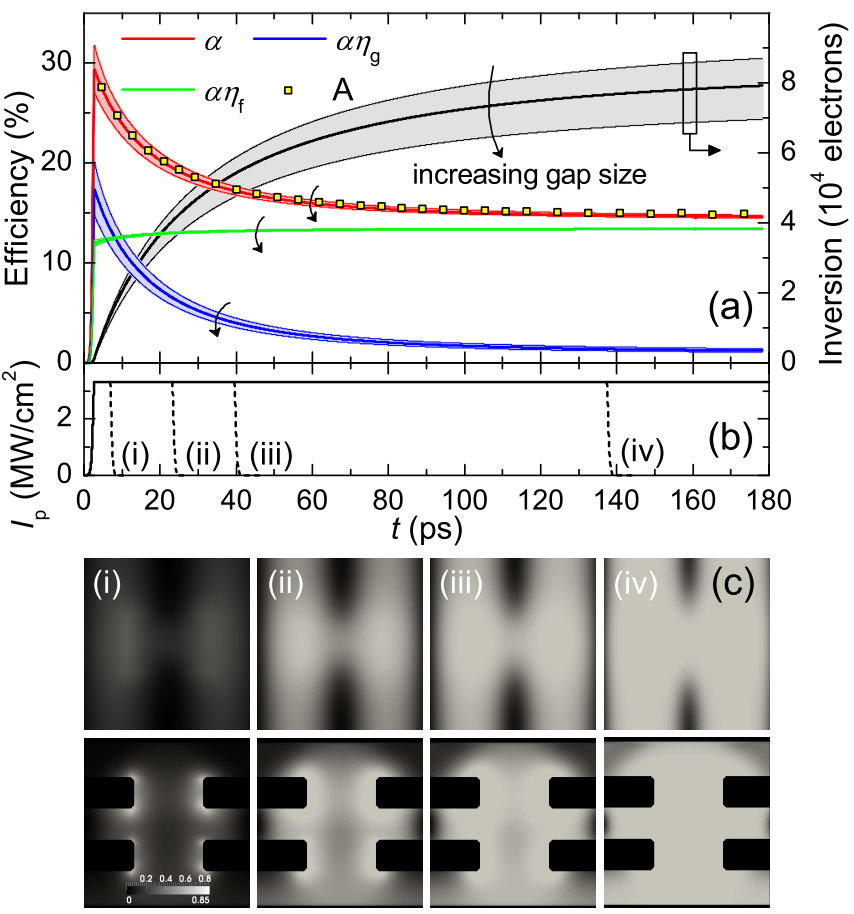

Figure 2. (color online) Conversion efficiencies over pump duration $\tau_{\mathrm{p}}$ for gap sizes of $0-10 \mathrm{~nm}$ (thick lines: $5 \mathrm{~nm}$ ). (a) Left axis: Total absorption efficiency $\alpha$ (red lines), external pump efficiency $\alpha \eta_{\mathrm{g}}$ (blue lines), and dissipative efficiency $\alpha \eta_{\mathrm{f}}$ (green lines); absorption coefficient $A$ (yellow squares) obtained from ATR calculations; Right axis: Total inversion $\left\langle N_{2}-N_{1}\right\rangle$ (black lines) of the gain material. (b) Pump intensity $I_{\mathrm{p}}$ over $\tau_{\mathrm{p}}$ indicating the switch-off times (dashed lines). (c) Spatial inversion profiles, $\left[N_{2}(\mathbf{r})-N_{1}(\mathbf{r})\right] / N$, for four different pump times (i)-(iv): (top row) $\mathrm{x}-\mathrm{y}$ cross section in the middle between the fishnet metal films; (bottom row) $\mathrm{x}-\mathrm{z}$ cross-section through the centers of the holes.

to metal surfaces, which we qualitatively accommodate for by considering a gap between the metal and the gain material with values of $g=0-10 \mathrm{~nm}$. The volume $V$ used for the rate retrieval [Eq. (2)] is chosen to tightly enclose the structure.

The amplification performance of the active doublefishnet critically depends on where and how efficiently energy is deposited in the gain medium and the metal during the pump process. While macroscopically one can only measure the total absorption, the presented methodology allows us to trace the conversion of energy into inversion and thermal energy microscopically within the gain medium and the metal films, as shown in Fig. 2. We pump the metamaterial with a pulse of constant peak intensity $I_{\mathrm{p}}=3.3 \mathrm{MW} / \mathrm{cm}^{2}$ but varying duration $\tau_{\mathrm{p}}=0-180 \mathrm{ps}$ (in steps of $1.38 \mathrm{ps}$ ) and record the gradual change in the efficiency with which energy is absorbed by the gain medium and the metal. To this end, we define the following photon conversion efficiencies: the total absoption efficiency $\alpha=-\langle\nabla \cdot \mathbf{S}\rangle /\left(I_{\mathrm{p}} A\right)$ is the net percentage of photons absorbed in the volume 
(influx); the internal pump efficiency $\eta_{\mathrm{g}}=\Gamma_{\mathrm{g}} / \Lambda$ (with $\left.\Gamma_{\mathrm{g}}=-\Gamma_{\mathrm{e}}-\Gamma_{\mathrm{a}}\right)$ is the percentage of absorbed photons transfering their energy to the gain medium; and the internal dissipative 'efficiency' $\eta_{\mathrm{f}}=-\Gamma_{\mathrm{f}} / \Lambda$ is the percentage of absorbed photons dissipated in the metal.

Figure 2(a) shows that just after the ramp-up of the pump pulse (2.7 ps) approximately a third of all incident photons are absorbed inside the cavity (red lines), with the efficiency of the energy transfer to the dye (blue lines) dominating over dissipative losses (green lines) by a factor of $\eta_{\mathrm{g}} / \eta_{\mathrm{f}} \approx 1.45$. The energy absorbed by the dye is converted into inversion, which initially concentrates around the metal interfaces where the pump field enhancement is strong [see Fig. 2(c) (i)]. As the duration of the pump increases, the efficiency with which inversion is created in the dye $\left(\alpha \eta_{\mathrm{g}}\right)$ decreases rapidly falling below the contribution from dissipative losses [Fig. 2(a)]. This drop in pump efficiency is caused by the saturation of the gain medium, with areas of high inversion gradually spreading out from close to the metal, as can be seen in Figs. 2(c) (i)-(iv). Eventually, a global saturation of the total inversion [Fig. 2(a), black lines] sets in where half the maximum value is already reached within the first 30 ps.

We further note that the efficiency with which energy is absorbed by the gain medium (blue lines) does not reach zero for $\tau_{\mathrm{p}} \rightarrow \infty$ since the broad emission line of the dye overlaps with the pump wavelength. As the medium approaches saturation the internal quantum yield decreases and the electric field energy is increasingly absorbed by the dye in absorption-emission cycles without creating inversion.

Having pumped the active metamaterial into inversion, we now probe it with a signal pulse of duration $\tau_{\mathrm{s}}=1 \mathrm{ps}$ and peak intensity $I_{\mathrm{s}}=1.23 \mathrm{~kW} / \mathrm{cm}^{2}$ (Fig. $3(\mathrm{~b})$, black line) at the emission wavelength of the dye. Note that the energy inside the resonator (Fig. 3(b), red line) does not instantaneously follow the excitation $I_{\mathrm{S}}$, but exhibits a rising flank during switch-on and an exponential decay after the switch-off of the probe pulse. After the transient build-up of energy in the resonant mode (Fig. 3(a), negative values for the black line) two distinct phases of constant rates follow: steady-state continuous excitation $(\mathrm{CE})$ characterized by $\Gamma_{\mathrm{t}}=0$; and free decay $(\mathrm{FD})$ of the excited mode with a $Q$-factor $Q=\omega_{\mathrm{e}} / \Gamma_{\mathrm{t}}$. Figure 3(a) exemplifies the retrieval of rates for a specific pump duration (and inversion) and $5 \mathrm{~nm}$ gap size. By performing corresponding probe experiments for pump times $\tau_{\mathrm{p}}=0-180 \mathrm{ps}$ and gap sizes $g=0-10 \mathrm{~nm}$ we obtain the results reported in Figs. 3(c) and (d).

In the CE phase [Fig. 3(c)], the energy inside the driven resonator does not change with time and, according to Eq. (2), the flux rate $\Lambda$ (red lines) then equals the difference between the net gain rate $\Gamma_{\mathrm{g}}$ (blue lines) and the dissipative-loss rate $\Gamma_{\mathrm{f}}$ (green lines), i.e. $\Lambda=\Gamma_{\mathrm{g}}-\Gamma_{\mathrm{f}}$. Figure 3(c) shows that overcoming the amplification
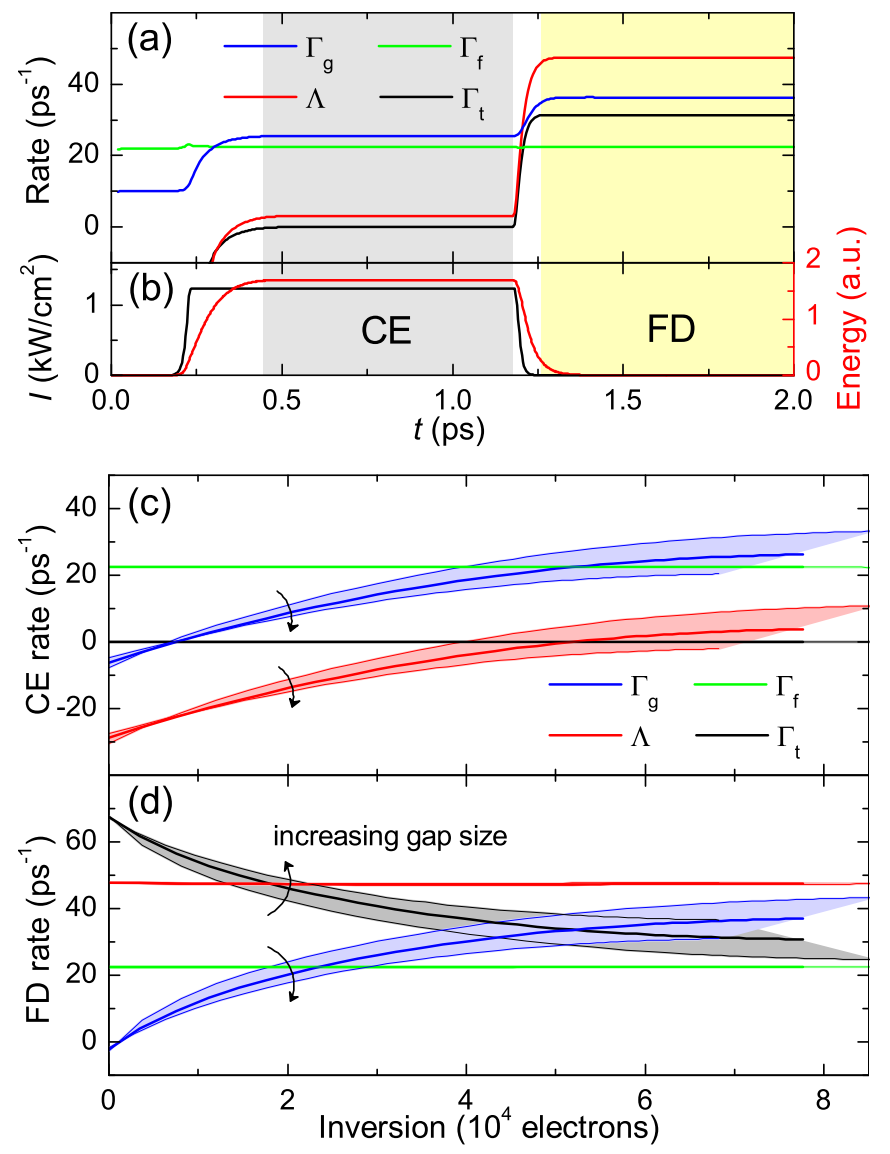

Figure 3. (color online) (a) Rate dynamics during probe process for $5 \mathrm{~nm}$ gap in amplifying regime: Gain rate $\Gamma_{\mathrm{g}}$ (blue line), dissipative loss rate $\Gamma_{\mathrm{f}}$ (green line), outflux rate $\Lambda$ (red line), and energy decay rate $\Gamma_{\mathrm{t}}$ (black line). (b) Probe pulse intensity $I_{\mathrm{s}}$ (black line) and energy $U$ inside the volume (red line) over time, with highlighted regimes of continuous excitation (CE) and free decay (FD). (c,d) Extracted rates over total inversion in the gain medium for gap sizes $0-10 \mathrm{~nm}$ under CE (c) and in FD (d) [colors as in (a)].

threshold $\Lambda=0$, where the gain rate equals the loss rate, requires higher values of total inversion for larger gap sizes. This is because the field of the resonator mode at the probe wavelength $\mathbf{E}\left(\lambda_{\mathrm{e}}, \mathbf{r}\right)$, which weighs the local gain rate according to $\Gamma_{\mathrm{g}} \propto \int \Gamma_{\mathrm{g}}(\mathbf{r})\left|\mathbf{E}\left(\lambda_{\mathrm{e}}, \mathbf{r}\right)\right|^{2} \mathrm{~d} V[16]$, is highest close to the metal. While the inversion in these regions makes larger contributions to the effective gain, its impact on the gain-enhancement is less strong than one might expect, making amplification possible even for larger gap sizes of up to $g \gtrsim 7.5 \mathrm{~nm}$. Energy transfer close to the metal interfaces (electron-hole coupling [9]) is therefore expected to increase the threshold but does not necessarily prevent amplification.

When the probe is switched off, the rates rebalance and enter the FD phase [see Fig. 3(d)]. The energy inside the resonator then decays exponentially with a rate $\Gamma_{\mathrm{t}}=$ $\Lambda+\Gamma_{\mathrm{f}}-\Gamma_{\mathrm{g}}>0$. Just as the amplification threshold was 


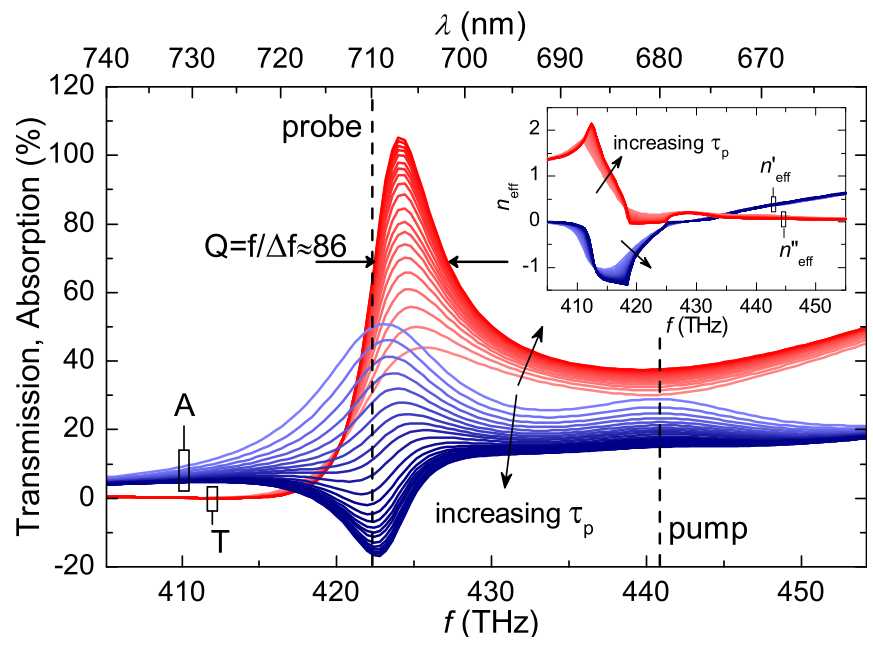

Figure 4. (color online) Absorption (blue lines) and transmission spectra (red lines) for pump times $\tau_{\mathrm{p}}=2.7-180 \mathrm{ps}$ (lighter to darker colors, black arrows) for $5 \mathrm{~nm}$ gap size. The $Q$-factor calculated from the width of the transmission resonance is given for $\tau_{\mathrm{p}}=180 \mathrm{ps}$. Inset: real (blue lines) and imaginary parts (red lines) of the effective refractive index retrieved from the complex ATR spectra.

characterized by $\Lambda=0$ in the CE regime, we identify the lasing threshold as $\Gamma_{\mathrm{t}}=0$ in the FD regime. Here, for lasing to occur the gain would have to overcome the sum of dissipative and radiative loss.

The constancy of the dissipative loss rate $\left(\Gamma_{\mathrm{f}} \approx\right.$ $22 \mathrm{ps}^{-1}$ ) and the radiative damping rate in free decay $\left(\Lambda \approx 48 \mathrm{ps}^{-1}\right)$ show that neither the overlap of the field with the metal nor the coupling to the radiative continuum are affected by gap size or inversion. This indicates that the mode profiles in the two regimes $(\mathrm{CE}$ and $\mathrm{FD}$ ) remain constant. Interestingly, the dissipative loss rate also remains unaltered during the $\mathrm{CE} \rightarrow \mathrm{FD}$ transition, despite the switch-off of the incident field. In effect, a window of amplification exists for the gain rate $\Gamma_{\mathrm{g}}$ between the point of loss-compensation and the lasing threshold, $\Gamma_{\mathrm{f}}<\Gamma_{\mathrm{g}}<\Gamma_{\mathrm{f}}+\Lambda^{\mathrm{FD}}$. For the considered active plasmonic metamaterial the radiative flux rate $\Lambda^{\mathrm{FD}}$ (Fig. 3 (d), red lines) is more than two times larger than the dissipative-loss rate $\Gamma_{\mathrm{f}}$ (green lines). In practice, the regime of amplification might be further limited by gain saturation as $\Gamma_{\mathrm{g}} \leq \Gamma_{\mathrm{g}}^{\mathrm{sat}}$. For sufficiently high gain densities the lasing threshold can be crossed. The energy inside the resonator then increases exponentially $\left(\Gamma_{t}<0\right.$, lasing instability) until gain depletion sets in, which is indeed observed.

The microscopically calculated cavity $Q$-factor and the amplification threshold can be related to the ATR spectra depicted in Fig. 4. We see that for longer pump durations (increased inversions) all spectra converge due to gain saturation, including those of the extracted effective refractive index [20] shown in the inset. No further increase (decrease) in the transmission (absorp- tion) of the probe pulse through the structure is possible. The width of the transmission resonance narrows to $\Delta f \approx 4.9 \mathrm{THz}$ at saturation, which is in good agreement with the extracted decay rate of $\Gamma_{\mathrm{t}} \approx 31 \mathrm{ps}^{-1} \approx 2 \pi \Delta f$ [last point of full black line in Fig. 3(d)]. Further, the absorption coefficient $A$ at the probe wavelength becomes zero at precisely the same inversion value at which $\Lambda=0$ in the $\mathrm{CE}$ regime [full red line in Fig. 3(c)]. At the pump wavelength, $A$ remains positive, decreases with higher values of inversion and, presented as yellow squares in Fig. 2, agrees quantitatively with the microscopically calculated absorption efficiency $\alpha$.

In conclusion, we have introduced a generic Maxwell Bloch-based methodology that allowed us to investigate the dynamic origins of light amplification, i.e. the transfer of energy between microscopic gain and loss channels, in a fishnet metamaterial. The extracted rates associated with the subsystems of dye, metal, and radiative continuum reveal that radiative damping dominates over the dissipative loss. Our results show that steady-state net amplification free of lasing instabilities is possible over a broad parameter regime and may be achieved even when gain passivation close to the metal is considered.

We gratefully acknowledge support by the Leverhulme Trust, the Royal Academy of Engineering and the EPSRC (UK).

*o.hess@imperial.ac.uk

[1] W. Cai and V. Shalaev, Optical metamaterials: Fundamentals and applications (Springer, Heidelberg, 2009).

[2] S. Zhang et al., Opt. Express 13, 4922 (2005); Phys. Rev. Lett. 95, 137404 (2005).

[3] A. Mary, S. G. Rodrigo, F. J. Garcia-Vidal, and L. Martin-Moreno, Phys. Rev. Lett. 101, 103902 (2008).

[4] J. Valentine et al., Nature (London) 455, 376 (2008).

[5] C. M. Soukoulis and M. Wegener, Science 330, 1633 (2010).

[6] S. Anantha Ramakrishna and J. B. Pendry, Phys. Rev. B 67, 201101 (2003).

[7] E. Plum, V. A. Fedotov, P. Kuo, D. P. Tsai, and N. I. Zheludev, Opt. Express 17, 8548 (2009).

[8] N. Meinzer et al., Opt. Express 18, 24140 (2010).

[9] I. De Leon and P. Berini, Nat. Photonics 4, 382 (2010).

[10] R. F. Oulton et al., Nature (London) 461, 629 (2009).

[11] S. Xiao et al., Nature (London) 466, 735 (2010).

[12] S. Wuestner, A. Pusch, K. L. Tsakmakidis, J. M. Hamm, and O. Hess, Phys. Rev. Lett. 105, 127401 (2010).

[13] A. Fang, T. Koschny, and C. M. Soukoulis, Phys. Rev. B 82, 121102 (2010).

[14] J. Yang, C. Sauvan, H. T. Liu, and P. Lalanne, Phys. Rev. Lett. 107, 043903 (2011).

[15] S. Wuestner, A. Pusch, K. L. Tsakmakidis, J. M. Hamm, and O. Hess, Phil. Trans. R. Soc. A 369, 3525 (2011).

[16] Y. Sivan, S. Xiao, U. K. Chettiar, A. V. Kildishev, and V. M. Shalaev, Opt. Express 17, 24060 (2009).

[17] K. Böhringer and O. Hess, Prog. Quantum Electron. 32, 159 (2008); Prog. Quantum Electron. 32, 247 (2008). 
[18] R. Ruppin, Phys. Lett. A 299, 309 (2002).

[19] J. A. McMahon et al., J. Phys. Chem. C 113, 2731 (2009).
[20] D. R. Smith, S. Schultz, P. Markoš, and C. M. Soukoulis, Phys. Rev. B 65, 195104 (2002). 